\title{
Análise da qualidade do serviço e do valor em eventos de esporte e atividade física na visão dos participantes
}

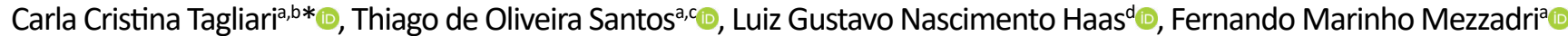

\section{Palavras Chave:}

Gestão da qualidade; Avaliação dos serviços; Atividades esportivas; Valor preditivo.

\begin{abstract}
RESUMO
Esta investigação pretende avaliar a percepção da qualidade do serviço em um evento esportivo público e o seu valor para a comunidade. Utilizou-se o modelo conceptual de Ko e Pastore (2005) para medir a qualidade do serviço. A amostra foi composta de 376 participantes. Recorreu-se à análise fatorial confirmatória e ao modelo de equações estruturais para análise dos dados. Ambos modelos de medida e estrutural indicaram boas qualidades psicométricas. Os resultados sugerem que a qualidade do serviço pode ser avaliada a partir das atividades ofertadas, qualificação dos profissionais, a interação entre os participantes e uma boa atmosfera. A percepção da qualidade mostrou um impacto positivo no valor do evento para comunidade.
\end{abstract}

\section{ABSTRACT}

This research aims to evaluate the perception of service quality in a public sports event and its value to the community. The conceptual proposal of Ko and Pastore (2005) was used to measure the quality of service. The sample consisted of 376 participants. We used confirmatory factorial analysis and the structural equations model to analyze the data. Both measurement and structural models indicated good psychometric qualities. The results have suggested that the quality of service could be evaluated from the activities offered, qualification of the professionals, the interaction between the participants and a good atmosphere in the event. In addition, the perception of quality has showed a positive impact on the value of the event for the community.

\section{RESUMEN}

Esta investigación, pretende evaluar la percepción de la calidad del servicio en un evento deportivo público y su valor para la comunidad. Se utilizó la propuesta conceptual de Ko y Pastore (2005) para medir la calidad del servicio. Un total de 376 individuos participaron en la investigación. Se utilizó un análisis factorial confirmatorio y el modelo de ecuaciones estructurales para el análisis. Los modelos indicaron buenas cualidades psicométricas. Los resultados sugieren que la calidad del servicio puede ser evaluada a partir de las actividades ofrecidas, calificación de los profesionales, interacción entre los participantes y una buena atmósfera en el evento. La percepción de la calidad impactó positivamente en el valor del evento para la comunidad.

\footnotetext{
a Universidade Federal do Paraná, Programa de Pós-graduação em Educação Física, Curitiba, PR, Brasil.

${ }^{b}$ Prefeitura Municipal de Curitiba, Curitiba, PR, Brasil.

' Universidade Europeia, Lisboa, Portugal.

${ }^{\mathrm{d}}$ Faculdade de Motricidade Humana da Universidade de Lisboa, Lisboa, Portugal.
}

\author{
*Autor correspondente: \\ Carla Cristina Tagliari \\ E-mail: carlatagliari@gmail.com
}

Recebido em 10 de julho de 2019; Aceito em 20 de agosto de 2019. 


\section{INTRODUÇÃO}

A qualidade do serviço é um elemento que deve ser observado na oferta de programas de esporte e atividade física (Chelladurai e Chang, 2000). Zeithaml (1988, p.03), define a qualidade do serviço como "o julgamento geral do consumidor sobre a excelência ou superioridade de um produto". Bitner e Hubbert (1994, p.77) apontam ser "a impressão geral do consumidor sobre o produto". Ambas definições evidenciam a perspectiva da percepção do consumidor como fator importante para o desenvolvimento de produtos e serviços. No contexto da oferta pública, um dos desafios está na proposição de serviços diferenciados e orientados para uma perspectiva eficiente de utilização de recursos e consequente qualidade (Carvalho e Brito, 2012). Kotler e Armstrong (2008) definem serviço como uma atividade ou benefício que uma organização oferece, não resultando da aquisição de um produto.

Ao longo do tempo, a análise da qualidade do serviço tem se constituído como prioridade dos governos visando satisfazer as necessidades da população (Carvalho e Brito, 2012; Roy e Seguin, 2000). No Brasil, programas de esporte e atividade física têm assumido historicamente uma posição de destaque no cenário das pesquisas (Becker et al., 2016; Bielemann et al., 2015). Nesta investigação entende-se o conceito de esporte tratado numa perspectiva "de atividade corporal que, através de participação ocasional ou organizada, visam exprimir ou melhorar a condição física e o bem-estar mental, constituindo relações sociais ou a obtenção de resultados" e atividade física como "a prática vinculada à promoção da saúde e elevação da qualidade de vida" (Brasil, 2015 p.15). A rápida expansão destes programas não tem sido acompanhada de uma compreensão sobre as características, os tipos de ações, implementação, resultados e outros aspectos que compõem estas iniciativas, bem como, o seu impacto para a comunidade (Becker et al., 2016). Desta forma, avaliar este tipo de programa pode ajudar a entender se atendem ou não as necessidades da população.

Chelladurai e Chang (2000) classificam como fundamental a análise da qualidade do serviço em programas de atividade física e/ou o esporte. Os estudos nesta perspectiva, tem tido como foco a identificação de dimensões para avaliar o cenário da qualidade do serviço (Ko e Pastore, 2005), a análise da qualidade do serviço em centros de fitness (Lam et al., 2005) ou a percepção da qualidade por participantes de programas públicos que fomentam a prática da atividade física (Nuviala et al., 2012). Diferentes instrumentos são propostos na literatura para avaliar a qualidade do serviço em esporte e atividade física
(Howat et al., 1996, Kim e Kim, 1995, Ko e Pastore, 2005). Assim, compreender a percepção dos participantes é uma importante fonte de informação para melhorar o serviço (Nuviala et al., 2012). Isto porquê, ações que objetivam o fomento ao esporte e a prática da atividade física tendem a impactar positivamente na melhoria da qualidade de vida da comunidade (Chelladurai e Chang, 2000). Por outro lado, uma percepção menos positiva da qualidade do serviço tende a diminuir a avaliação do quanto este evento é importante na vida dos cidadãos. Deste modo, o objetivo deste estudo foi avaliar a percepção dos participantes de um evento de esporte e atividade física sobre a qualidade do serviço e o consequente impacto na percepção de valor para o participante e para a comunidade.

\section{Conceptualização do modelo}

Para o presente estudo, optou-se por adaptar o instrumento "Scale of Service Quality in Recreational Sport (SSQRS)" proposto por Ko e Pastore (2005), para avaliar as percepções dos participantes sobre a qualidade do serviço em um evento. A adoção desta proposta conceptual foi feita com base na: a) adequação ao contexto do estudo; b) enquadramento da proposta ao tipo de evento analisado; c) ser uma escala desenvolvida com boas qualidades psicométricas e já testada. Esta escala inclui as dimensões brevemente descritas a seguir.

\section{Qualidade das atividades no evento}

Percepção baseada na experiência com o evento, medida através da variedade, da atratividade e da conveniência dos horários das atividades, bem como, a facilidade em se obter informações atualizadas sobre os serviços ofertados. Quanto mais conveniente e adequada são as atividades vivenciadas, melhor é a percepção sobre a qualidade do evento. Por outro lado, questões como a falta de informação sobre as atividades tendem a diminuir esta percepção (Ko e Pastore, 2005).

\section{Qualidade da interação}

Percepção subjetiva do serviço entregue, a partir das interações que ocorrem durante o encontro de serviço com os profissionais envolvidos no evento. É medida através da percepção das atitudes, da especialização e familiaridade com o serviço, do desempenho no trabalho e da capacidade de resolver problemas (Howat et al., 1996; Wright et al., 1992). Estudos prévios apontam que o comportamento do profissional envolvido no evento pode alterar a percepção do participante sobre o serviço (Biscaia et al., 2017). 


\section{Qualidade da experiência}

Benefícios percebidos pelo participante quando participam em atividades (e.g., melhoria da condição física, habilidade, emoção e prazer). Ko e Pastore (2005) propõem medir esta dimensão a partir da valência (i.e., avaliação do participante sobre o resultado do evento para si), da sociabilidade (i.e., experiências positivas advindas da socialização) e da experiência social (i.e., resultado da participação esportiva). Assim, quanto melhor é a percepção sobre a experiência no evento, maior é a possibilidade de uma avaliação positiva da qualidade do serviço.

\section{Qualidade do ambiente físico}

Percepção do participante quanto a condição do ambiente de serviço (e.g., temperatura, iluminação, ruído, música). Observa-se o design da instalação incluindo componentes funcionais e estéticos do ambiente. Ko e Pastore (2005) apontam que uma percepção positiva do ambiente tende a influenciar positivamente a avaliação da qualidade do serviço. Do contrário, um ambiente com componentes funcionais e estéticos problemáticos tende a diminuir esta percepção.

Complementarmente, nesta investigação o impacto destas dimensões para a comunidade é medido pela percepção do valor do evento para o participante e para a comunidade, definido como a razão dos benefícios percebidos pelos participantes em relação ao investimento necessário para a sua participação no evento e/ou para a organização do evento (Biscaia et al., 2017).

\section{PROCEDIMENTOS METODOLÓGICOS}

Esta pesquisa foi realizada através de um procedimento em multietapas. Primeiro, uma revisão por especialistas dos itens propostos para a qualidade do serviço foi conduzida. Em seguida, uma avaliação empírica da escala através da análise fatorial confirmatória (AFC) e uma análise ao modelo de equações estruturais (MEE) foram realizadas para testar a relação da percepção da qualidade do serviço e do valor do evento.

\section{Instrumento}

Com base na conceptualização original de Ko e Pastore (2005), foi gerado um conjunto inicial de 16 itens para avaliar as quatro dimensões da qualidade do serviço. A dimensão da qualidade das atividades do evento incluiu quatro itens que se referem à: variedade de atividades no evento, informações disponibilizadas para os participantes e possibilidades de contato com a organização do evento. Já os itens da qualidade da interação referem-se à: eficiência da interação com os profissionais no evento, a rapidez e o nível de excelência da performance dos profissionais para resolver demandas dos participantes. Para medir a qualidade da experiência, outros quatro itens envolvem: sensação de bem-estar, sentimento positivo e atmosfera percepcionada pelo participante no evento. Por fim, para medir a qualidade do ambiente físico foram utilizados quatro itens para capturar a percepção do participante sobre: a estética, o conforto e a limpeza e conservação do local.

Com o objetivo de avaliar a validade preditiva da qualidade do serviço, o questionário final contou com medidas adicionais de valor do evento para o participante e para a comunidade. Três itens foram adaptados de Biscaia et al. (2017) para medir o valor hedônico (i.e., aspectos festivos do evento que refletem potencial entretenimento e ganho emocional para o participante na medida em que considera o valor investido para participar no evento). Outros três itens propostos pelos mesmos autores, foram usados para medir o valor simbólico (i.e., percepção do prestígio do evento para a comunidade, refletido pelo seu papel simbólico de melhoria a partir do serviço). Todos os itens foram medidos usando uma escala do tipo-Likert de sete pontos variando de "discordo totalmente" (1) a "concordo totalmente" (7).

\section{Procedimentos}

Antes da aplicação do instrumento recorreu-se à técnica de retrotradução, para garantir a precisão entre a escala original, na língua inglesa, e a versão dos itens para o português (Banville et al., 2000). Foram realizadas reuniões com gestores da Secretaria Municipal do Esporte, Lazer e Juventude (SMELJ) da cidade de Curitiba para alinhar o apoio para realizar a pesquisa. Seguidamente, para verificar a relevância e clareza dos itens para o contexto brasileiro foi realizada uma validação de conteúdo por três investigadores de gestão do esporte e posteriormente, um pré-teste com 20 professores de educação física, com o questionário composto por todos os itens do estudo e questões sociodemográficas. Após estas etapas foram sugeridas alterações na redação de quatro itens relacionados as dimensões da qualidade do serviço. Por fim, a versão final do instrumento foi considerada adequada para aplicação.

\section{Contexto do estudo e participantes}

Os dados foram coletados durante dois dias em um evento promovido pela SMEL no ano de 2018. Os 
pesquisadores abordaram os participantes no evento, explicando os objetivos da pesquisa. Em seguida, os que aceitaram voluntariamente responder ao questionário assinaram um termo de consentimento que garantia a confidencialidade dos dados.

Um total de 405 questionários foram respondidos. Questionários incompletos ou que possuíam mais de oito respostas repetidas em sequência foram eliminados da amostra final. Um total de 376 questionários foram utilizados para a análise. A idade média dos participantes foi de 35.30 anos $( \pm 11.85)$, sendo $53,3 \%$ dos participantes do gênero masculino e $46.7 \%$ do gênero feminino. Quanto ao grau de instrução, $62.6 \%$ declararam ter ensino superior. Sobre a participação em edições anteriores do evento apenas $25.5 \%$ já haviam participado. Um total $81.4 \%$ responderam não participar em algum programa regular, gratuito e público de esporte e atividade física e $77.7 \%$ praticam atividade física regularmente. Por fim, $97.9 \%$ indicaram apoiar a oferta de eventos públicos e gratuitos de esporte e atividade física.

\section{Análise dos dados}

Os dados foram analisados através do software SPSS (v.24.0) e AMOS (v.24.0). A caracterização da amostra foi feita com recurso à estatística descritiva. Avaliou-se as qualidades psicométricas dos modelos de medida através da análise fatorial confirmatória (AFC). $O$ ajuste aos dados foi assumido a partir da relação do $\chi^{2}$ (qui-quadrado) para os seus graus de liberdade inferior a 3.0 (Hair et al., 2009), dos valores de CFI (comparative-of-fit-index), o GFI (goodness-offit-index) e o TLI (Ticker-Lewis Index) maiores do que .90 (Hair et al., 2009) e do RMSEA (root mean square error of approximation) abaixo de .08 (Byrne, 2016). Estimou-se a consistência interna através da fiabilidade compósita (FC) com valores acima de .60 (Bagozzi e Yi, 1988). Avaliou-se a validade convergente por meio da variância extraída média (VEM) e valores próximos ou superiores a .50 foram considerados indicativos de boa validade convergente (Fornell e Larcker, 1981). A validade discriminante foi estabelecida para valores de correlações abaixo de .85 (Kline, 2005). Foram comparados o ajuste dos modelos de primeira e de segunda ordem através dos valores de Critérios de Informação de Akaike (CIA) e os valores Esperados do Índice de Validação Cruzada (EIVC) para determinar o modelo com melhor ajuste aos dados (Cronin et al., 2000). Através do modelo de equações estruturais (MEE), avaliou-se a capacidade explicativa do modelo causal pelos coeficientes de trajetória $(\beta)$ e de $\mathrm{R}^{2}$ (Hair et al., 2009), para um nível de significância de $p \leq .05$.

\section{RESULTADOS}

A análise dos dados dividiu-se em duas sessões: (a) avaliação do modelo de medida e (b) modelo estrutural.

\section{Avaliação do modelo de medida}

Testou-se a validade do modelo de medida para os fatores de primeira e segunda ordem da qualidade do serviço (i.e., qualidade das atividades do evento, qualidade da interação, qualidade da experiência e qualidade do ambiente físico).

Quatro itens das dimensões da qualidade do serviço apresentaram problemas quanto à normalidade, dois com valores de curtose acima de 7.0 e dois com pesos fatoriais abaixo de .50 (Ver Tabela 1). Devido à natureza empírica desta análise, os itens foram eliminados (Yoshida e James, 2011). Os demais itens não apresentaram problemas de normalidade com valores de assimetria variando de -0.95 a -2.01 e valores de curtose variando de .49 a 2.89 .

Após o refinamento da escala, um total 12 itens foi utilizado para a análise das dimensões da qualidade do serviço (Ver Tabela 1). O peso fatorial dos itens esteve acima de .50 indicando que cada item satura adequadamente na sua dimensão. A consistência interna das medidas foi aceita com os valores de fiabilidade compósita acima de 70 e a VEM com valores próximos de .50 indicando validade convergente para os constructos (Ver Tabela 1). A matriz de comparação entre os valores da VEM das dimensões e a correlação destas ao quadrado sugerem valores abaixo de .85 indicando a validade discriminante para o modelo de primeira ordem.

Os resultados da AFC para o modelo de primeira ordem $[\chi 2(48)=176.96(p<.01) ; \chi 2 / d f=3.68 ; C F I=.93$; $\mathrm{GFI}=.93 ; \mathrm{TLI}=.91 ; \mathrm{RMSEA}=.08$ ] indicaram um ajuste aceitável aos dados. Embora o valor de $\chi 2 / \mathrm{df}$ exceda o valor de referência os valores de CFI, GFI e TLI foram maiores que .90, critério para bom ajuste (Hair et al., 2009). Além disso, o valor de RMSEA indica um ajuste aceitável aos dados (Byrne, 2016).

A qualidade de ajuste do modelo de segunda ordem indicou um bom ajuste aos dados $[\chi 2(50)=179.87(\mathrm{p}<.01) ; \chi 2 / \mathrm{df}=3.59 ; \mathrm{CFI}=.94 ; \mathrm{GFI}=.92$; $\mathrm{TLI}=.91 ; \mathrm{RMSEA}=.08]$. Uma análise dos coeficientes de trajetória indica que todas as dimensões de primeira ordem estavam significativamente relacionadas com a dimensão de segunda ordem da qualidade do serviço (Ver Figura 1). Os valores de CIA e EIVC indicaram diferenças entre o modelo de primeira ordem $(C I A=236.96$; EIVC=.63) e segunda ordem da 
Tabela 1. Modelo de medida: Pesos fatoriais (PF); Fiabilidade compósita (FC); variância extraída média (VEM).

\begin{tabular}{|c|c|c|c|}
\hline Dimensões/Itens & PF & FC & VEM \\
\hline Qualidade das atividades do evento & & .70 & .44 \\
\hline O evento tem um número variado de atividades & .69 & & \\
\hline É fácil de contactar a organização do evento através de um site & .76 & & \\
\hline Informações atualizadas estão disponíveis na mídia sobre o evento e suas atividades & .66 & & \\
\hline \multicolumn{4}{|l|}{ O horário de funcionamento das atividades do evento é conveniente ${ }^{a}$} \\
\hline Qualidade da interação & & .85 & .65 \\
\hline Os profissionais no evento estão prontos para agir se ocorrer qualquer problema & .80 & & \\
\hline No evento os profissionais lidam com problemas de forma rápida e satisfatória & .85 & & \\
\hline No evento os profissionais lidam efetivamente com as necessidades de cada um dos participantes & .79 & & \\
\hline Os profissionais no evento parecem conhecer muito bem as suas funções ${ }^{b}$ & .79 & & \\
\hline Qualidade da experiência & & .76 & .51 \\
\hline Eu me sinto bem com o que acontece no evento & .69 & & \\
\hline Eu tenho um bom sentimento ao participar do evento & .72 & & \\
\hline Eu realmente gosto do clima criado no evento & .74 & & \\
\hline \multicolumn{4}{|l|}{ Eu avalio de forma positiva os resultados das atividades realizadas no evento ${ }^{b}$} \\
\hline Qualidade do ambiente físico & & .79 & .55 \\
\hline O local do evento é esteticamente atraente & .76 & & \\
\hline O local do evento é confortável & .73 & & \\
\hline O local onde o evento é realizado é limpo e bem conservado & .73 & & \\
\hline \multicolumn{4}{|l|}{ O local do evento é seguro ${ }^{a}$} \\
\hline Valor Hedônico & & .86 & .67 \\
\hline $\begin{array}{l}\text { Considerando a experiência que tive no evento, o dinheiro que gastei (e.g. deslocamentos, alimentação) } \\
\text { para participar é justo }\end{array}$ & .76 & & \\
\hline $\begin{array}{l}\text { O dinheiro que investi para participar no evento (e.g. deslocamentos, alimentação) é adequado } \\
\text { para a experiência que vivi }\end{array}$ & .88 & & \\
\hline No evento o dinheiro que gasto para vir e participar no evento é bem gasto & .82 & & \\
\hline Valor Simbólico & & .79 & .66 \\
\hline Como cidadão, sinto-me orgulhoso em ter um evento como este & .77 & & \\
\hline Eu acredito que este evento melhora a imagem da cidade & .85 & & \\
\hline O investimento neste evento torna a cidade mais próspera ${ }^{b}$ & & & \\
\hline
\end{tabular}

Fonte: dados da pesquisa conduzida pelos autores. Notas: altens eliminados, valores de curtose acima do limiar de 7.0; ${ }^{b}$ Itens eliminados, apresentaram pesos fatoriais abaixo de .50 .

qualidade do serviço ( $\mathrm{Cl} A=235.87$; EIVC=.62). Com base na proposta conceptual de Ko e Pastore (2005) e o resultado da comparação entre os modelos optou-se por utilizar o modelo de segunda ordem para análises subsequentes por apresentar um ajuste mais parcimonioso aos dados. Em seguida, avaliou-se a validade preditiva da qualidade do serviço no valor do evento para a comunidade.

\section{Modelo estrutural}

Uma AFC mostrou que um item do valor simbólico apresentou um peso fatorial abaixo de .50 e este foi eliminado (Fornell e Larcker, 1981). Os índices de ajustamento para o modelo de medida com as dimensões da qualidade do serviço ( $F C=.92$; VEM=.73), do valor simbólico e do valor hedônico $\left[\chi^{2}(112)=317.13(p<.01) ; \chi^{2} / g l=2.83 ; C F I=.94 ; G F I=.91\right.$; $\mathrm{TLI}=.92 ; \quad \mathrm{RMSEA}=.07]$ e $\mathrm{O} \operatorname{MEE} \quad\left[\chi^{2}(113)=364.96\right.$ $(p<.01) ; \quad \chi^{2} / g l=3.23 ; \quad C F I=.92 ; \quad G F I=.90 ; \quad T L I=.91 ;$ RMSEA=.07] indicaram um bom ajustamento aos dados (Ver Tabela 1).

A dimensão da qualidade do serviço na percepção dos participantes mostrou um efeito positivo e significativo no valor simbólico explicando $66 \%$ da sua variância, e no valor hedônico explicando $61 \%$ desta relação. 


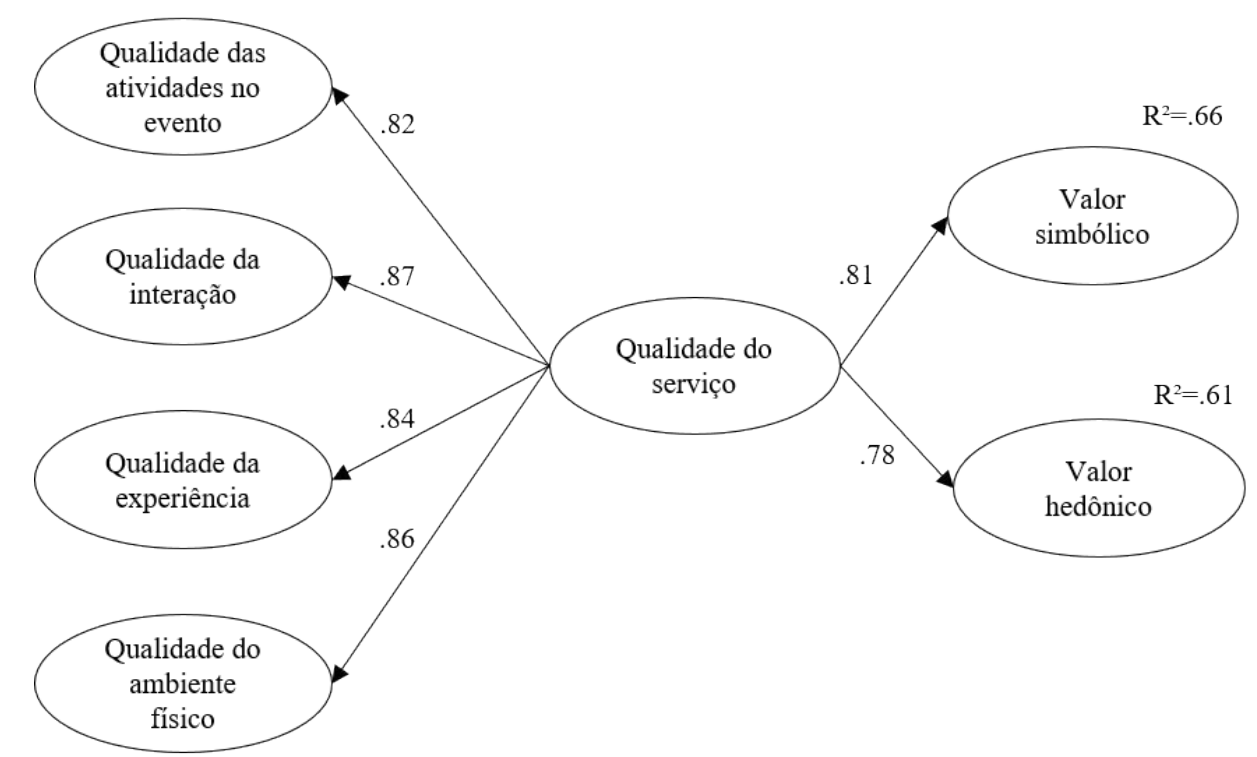

Figura 1. Modelo estrutural. Fonte: Dados da pesquisa conduzida pelos autores.

\section{DISCUSSÃO}

Esta investigação teve por objetivo avaliar a percepção dos participantes de um evento de esporte e atividade física sobre a qualidade do serviço e o valor para a comunidade através da proposta teórica de Ko e Pastore (2005). Os resultados apontam para uma influência positiva da dimensão da qualidade das atividades no evento (i.e., a variedade, atratividade e informações disponíveis sobre as atividades ofertadas e a conveniência dos horários), na percepção da qualidade do serviço. Estudos prévios (Nuviala et al., 2012; Lam et al., 2005) apontam que uma boa percepção da qualidade do serviço está relacionada aos interesses dos participantes. Assim, avaliações sobre as preferências dos participantes sobre atividades ofertadas podem potenciar a percepção da qualidade do serviço. Igualmente, o desenvolvimento de atividades voltadas a interação e ao bem-estar dos participantes fazem com que estes percebam de forma positiva o serviço no evento (Morales e Sánchez, 2003; Ruiz, 2011).

Complementarmente, os resultados desta pesquisa sugerem que a interação com os profissionais no evento é um componente importante da qualidade do serviço. Estudos apontam que questões como a atitude, experiência e capacidade da equipe de trabalho constituem-se parte fundamental da experiência no evento (Getz et al., 2001; Biscaia et al., 2017). Neste sentido, questões como disponibilidade, empatia, atitudes positivas e familiaridade dos profissionais no desenvolvimento das ações tendem a interferir positivamente na percepção do consumidor sobre o serviço. Assim, o aprimoramento da intervenção dos profissionais, através da formação e do conhecimento técnico deve ser considerado pelos gestores responsáveis por este tipo de evento.

Os resultados deste estudo sugerem que a qualidade da experiência no evento, que se refere aos benefícios advindos da participação e da socialização no evento influenciam a percepção do participante da qualidade do serviço. Estudos prévios (Ko et al., 2011; Brady e Cronin, 2001) destacam a relação de gratificação social de estar com outras pessoas que usufruem da mesma atividade e que, esta oportunidade de interação se positiva, tende a aumentar as chances de uma boa percepção quanto a qualidade do serviço. Assim, desenvolver atividades que favoreçam a comunicação, reforcem a ideia de grupo e fomentem a interação com outros participantes, pode ser importante para aumentar a qualidade do evento.

Complementarmente, os resultados da percepção do participante sobre a qualidade do ambiente demonstram que as questões relativas à limpeza, iluminação, esquema de cores e organização de estruturas e materiais para as atividades, são um fator importante na percepção positiva da qualidade do serviço (Voon et al., 2014). Deste modo, gestores de eventos no contexto do esporte e atividade física devem ter atenção na criação de um ambiente físico esteticamente atrativo com materiais adequados a prática. Especificamente neste estudo, os dados foram coletados em um parque onde o evento era realizado. Assim, pensar o design e a adaptação dos locais do evento é um fator importante para a entrega de um melhor serviço e consequente avaliação positiva da sua qualidade.

Adicionalmente, esta investigação propôs medir o impacto da percepção da qualidade do serviço sobre 
o valor do evento (Biscaia et al., 2017). O valor pode ser interpretado, de forma global, como resultado da avaliação da utilidade do serviço, baseado na relação entre benefícios obtidos e recursos, como tempo e esforço, empregados pelo participante (Yoshida et al., 2013). De acordo com os resultados deste estudo, pode-se argumentar que melhorias no orgulho da comunidade (Inoue e Havard, 2014) ou nos benefícios individuais advindos da participação em eventos como o estudado, possuem uma relação direta com a percepção positiva da qualidade do serviço (Heere et al., 2013).

Os resultados do modelo estrutural sugerem que questões como uma boa organização das atividades no evento, a qualificação dos profissionais, a interação com outros participantes e a estrutura física do local de realização do evento impactam na percepção de valor hedônico e simbólico. Dado que o valor hedônico está relacionado ao benefício afetivo e experiencial percebido pelo participante (Yoshida et al., 2013), gestores de eventos devem reforçaralgumas estratégias como: adicionar adereços para melhorar o ambiente e a atratividade ou criar oportunidades sociais para que todas as pessoas possam "viver o evento" fora do seu espaço (Biscaia et al., 2017). Além disso, decisões como a de instalar em locais estratégicos sinalização sobre os eventos, telões e oferecer lembranças da participação no evento também podem ser medidas interessantes para melhorar a atmosfera do evento e elevar a percepção do valor hedônico.

O papel preditivo da qualidade do serviço no valor simbólico sugere que gestores devem criar uma atmosfera atraente para o evento contribuindo assim para aumentar as percepções dos participantes sobre os benefícios do evento para a comunidade. Portanto, a realização de um evento esportivo deve representar uma oportunidade para o desenvolvimento de programas e infraestrutura que permitam o aumento da boa atmosfera do evento e promovam benefícios futuros e duradouros não relacionados ao esporte como: maior orgulho da comunidade, melhoria dos serviços de segurança, transportes, saúde entre outros (Biscaia et al., 2017).

Os resultados desta investigação também suportam a ideia de que a experiência com outros participantes, tem efeito tanto no valor hedônico quanto no simbólico. A experiência coletiva causa certo entusiasmo em quem está no evento, o que de certa forma aumenta a percepção da importância do evento para o aumento do sentido de pertencimento a comunidade agregando um valor pessoal importante aos participantes (Yoshida et al., 2013). Deste modo, embora os participantes empreguem um determinado valor financeiro para participar em eventos como o estudado, os benefícios percebidos na participação no evento são maiores.

Também é importante notar que os aspectos funcionais do serviço (e.g.) estrutura física, comunicação do evento) contribuem para diminuir a percepção sobre o custo-benefício em participar no evento, ou seja, quanto melhor é a percepção da qualidade, maior é a percepção do benefício que se tem em participar no evento (Yoshida et al., 2013). Assim, uma avaliação prévia da experiência que será vivenciada no evento (e.g., definição de local mais adequado ou uma comunicação/divulgação efetiva do evento) tendem a auxiliar na criação de uma melhor experiência e consequente benefício do evento para si e para a comunidade.

Como visto, a avaliação da percepção dos participantes de um evento de esporte e atividade física sobre a qualidade do serviço e o impacto na percepção de valor pode auxiliar gestores a melhorar os processos de criação e gestão deste tipo de evento.

\section{CONCLUSÕES}

Este estudo representa um esforço inicial para fornecer informações relativas à percepção da qualidade de eventos de esporte e atividade física na percepção dos participantes, fornecendo subsídios aos gestores quanto a compreensão de elementos (e.g., qualidade das atividades no evento, da interação, da experiência e do ambiente físico) que contribuam para a proposição e gestão de eventos culminando numa maior assertividade dos resultados.

Ainda assim, esta investigação apresenta limitações que devem ser consideradas em pesquisas futuras. Os dados foram coletados em apenas uma edição do evento em um único local. Outros estudos empíricos em locais e eventos diferentes podem ampliar a visão quanto a elementos importantes da gestão de eventos e em uma amostra mais diversificada. Além disso, a inclusão de outras variáveis no modelo de análise da qualidade do serviço (e.g., expectativas préevento) podem auxiliar num melhor planejamento da experiência dos participantes. Complementarmente, sugere-se que outras investigações sejam realizadas buscando a avaliação em programas de esporte e atividade física promovidos por entes públicos no Brasil.

Por fim, o conhecimento das dimensões da qualidade apresentadas nesse estudo deve contribuir para que os gestores possam atender as demandas dos participantes e proporcionar experiências de excelência a partir de aspectos que podem ser controlados e manipulados para melhorar a prestação 
de serviços. Tomar decisões sintonizadas com a realidade da comunidade aumenta a percepção da qualidade do evento e do seu valor. Isso permite concluir que é fundamental que sejam garantidas experiências positivas e previamente planejadas, a fim de assegurar melhores avaliações nos atributos da qualidade do evento. Deste modo, gestores públicos tendem a dar suporte a melhoria da qualidade de vida dos cidadãos e consequentemente na comunidade.

\section{APOIO FINANCEIRO}

O presente trabalho foi realizado com apoio da Coordenação de Aperfeiçoamento de Pessoal de Nível Superior Brasil (CAPES) - Código de Financiamento 001". "This study was financed in part by the Coordenação de Aperfeiçoamento de Pessoal de Nível Superior - Brasil (CAPES) Finance Code 001.

\section{AGRADECIMENTOS}

Secretaria Municipal do Esporte, Lazer e Juventude de Curitiba.

\section{REFERÊNCIAS}

Bagozzi RP, Yi Y. On the evaluation of structural equation models. Journal of the academy of marketing science. 1988 Mar 1;16(1):74-94.

Banville D, Desrosiers P, Genet-Volet Y. Translating questionnaires and inventories using a cross-cultural translation technique. Journal of teaching in Physical Education. 2000 Apr;19(3):374-87.

Becker L, Gonçalves P, Reis R. Programas de promoção da atividade física no Sistema Único de Saúde brasileiro: revisão sistemática. Revista Brasileira de Atividade Física \& Saúde. 2016 Mar 1;21(2):110-22.

Bielemann RM, da Silva BG, Coll CD, Xavier MO, da Silva SG. Impacto da inatividade física e custos de hospitalização por doenças crônicas. Revista de Saúde Pública. 2015 Jan 1;49:75-.

Biscaia R, Correia A, Santos T, Ross S, Yoshida M. Service quality and value perceptions of the 2014 FIFA World Cup in Brazil. Event Management. 2017 May 11;21(2):201-16.

Bitner MJ, Hubbert AR. Encounter satisfaction versus overall satisfaction versus quality. Service quality: New directions in theory and practice. 1994;34(2):72-94.

Brady MK, Cronin Jr JJ. Some new thoughts on conceptualizing perceived service quality: a hierarchical approach. Journal of marketing. 2001 Jul;65(3):34-49.

Brasil. Avaliação de efetividade de programas de atividade física no Brasil. Ministério da saúde, Brasília,2011, Disponível em: <http://bvsms.saude.gov.br/bvs/publicacoes/avaliacao_ efetividade_programas_atividade_fisica.pdf $>$. Acesso em: 12 out. 2018.

Byrne BM. Structural equation modeling with AMOS: Basic concepts, applications, and programming. Routledge, 2016.

Carvalho C, Brito C. Assessing users' perceptions on how to improve public services quality. Public Management Review. 2012 May 1;14(4):451-72.
Chelladurai P, Chang K. Targets and standards of quality in sport services. Sport Management Review. 2000 May 1;3(1):1-22.

Cronin Jr JJ, Brady MK, Hult GT. Assessing the effects of quality, value, and customer satisfaction on consumer behavioral intentions in service environments. Journal of retailing. 2000 Jun 1;76(2):193-218.

Fornell C, Larcker DF. Structural equation models with unobservable variables and measurement error: Algebra and statistics. 1981.

Getz D, O'Neill M, Carlsen J. Service quality evaluation at events through service mapping. Journal of travel research. 2001 May;39(4):380-90.

Hair JF. et al. Multivariate data analysis $7^{\text {th }}$ ed. New York, Pearson Prentice Hall, 2009.

Heere B, Walker M, Gibson H, Thapa B, Geldenhuys S, Coetzee $W$. The power of sport to unite a nation: The social value of the 2010 FIFA World Cup in South Africa. European Sport Management Quarterly. 2013 Sep 1;13(4):450-71.

Howat G, Absher J, Crilley G, Milne I. Measuring customer service quality in sports and leisure centres. Managing leisure. 1996 Jan 1;1(2):77-89.

Inoue $Y$, Havard CT. Determinants and consequences of the perceived social impact of a sport event. Journal of Sport Management. 2014 Maio;28(3):295-310.

Kim D, Kim SY. QUESC: An instrument for assessing the service quality of sport centers in Korea. Journal of sport management. 1995 May;9(2):208-20.

Kline RB. Methodology in the social sciences: Principles and practice of structural equation modeling. New York, NY. 2005.

Ko YJ, Pastore DL. A Hierarchial Model of Service Quality for the Recreational Sport Industry. Sport Marketing Quarterly. 2005 Jun 1;14(2).

Jae Ko Y, Zhang J, Cattani K, Pastore D. Assessment of event quality in major spectator sports. Managing Service Quality: An International Journal. 2011 May 17;21(3):304-22.

Kotler P; Armstrong G. Principles of marketing. $12^{\text {th }}$ ed. New Jersey, Person Prentice Hall, 2008.

Lam ET, Zhang JJ, Jensen BE. Service Quality Assessment Scale (SQAS): An instrument for evaluating service quality of health-fitness clubs. Measurement in physical education and exercise science. 2005 Jun 1;9(2):79-111.

Morales Sánchez, V. Evaluación psicosocial de la calidad en los servicios municipales deportivos; aportaciones desde el análisis de variabilidad. Tese (doutorado) - Universidad de Málaga, 2003.

Nuviala Nuviala $A$, Pérez-Ordás $R$, Boceta Osuna $M$, GraoCruces A, Nuviala Nuviala R, González Jurado JA. Calidad, satisfacción y valor percibido de los usuarios de un servicio deportivo público. Movimento. 2012;18(4).

Roy C, Seguin F. The institutionalization of efficiency-oriented approaches for public service improvement. Public Productivity \& Management Review. 2000 Jun 1:449-68.

Ruiz PG. Cuestionario para evaluar la calidad de servicios deportivos: estudio inicial de las propiedades psicométricas. Tese (doutorado) - Departamento de Fisiología y Educación Física y Deportiva, Facultad de Medicina, Málaga, 2011.

Ho Voon B, Lee N, Murray D. Sports service quality for event venues: evidence from Malaysia. Sport, Business and Management: An International Journal. 2014 May 6;4(2):125-41. 
Wright BA, Duray N, Goodale TL. Assessing perceptions of recreation center service quality: an application of recent advancements in service quality research. Journal of Park and Recreation Administration. 1992;10(3):33-47.

Yoshida M, James JD. Service quality at sporting events: Is aesthetic quality a missing dimension? Sport Management Review. 2011 Feb 1;14(1):13-24.
Yoshida M, James JD, Cronin JJ. Value creation: Assessing the relationships between quality, consumption value and behavioural intentions at sporting events. International Journal of Sports Marketing and Sponsorship. 2013 Jan 1;14(2):51-73. ZeithamI VA. Consumer perceptions of price, quality, and value: a means-end model and synthesis of evidence. Journal of marketing. $1988 \mathrm{Jul} ; 52(3): 2-2$. 